\title{
Evaluation of effect of tween 80 on characteristics of tadalafil $0.1 \%$ suspension
}

\author{
Majid Saeedi ${ }^{1,2^{*}}$, Mohammad Reza Rafati ${ }^{3}$, Katayoun Morteza-Semnani ${ }^{4}$, Atieh Yazdani Rostam ${ }^{5}$, \\ Hamid Reza Kelidari \\ ${ }^{1}$ Department of Pharmaceutics, Faculty of Pharmacy, Mazandaran University of Medical Sciences, Sari, Iran \\ ${ }^{2}$ Pharmaceutical Sciences Research Centre, Mazandaran University of Medical Sciences, Sari, Iran \\ ${ }^{3}$ Department of Clinical Pharmacy, Faculty of Pharmacy, Mazandaran University of Medical Sciences, Sari, Iran \\ ${ }^{4}$ Department of Medicinal Chemistry, Faculty of Pharmacy, Mazandaran University of Medical Sciences, Sari, Iran \\ ${ }^{5}$ Student Research Committee, Mazandaran University of Medical Sciences, Sari, Iran
}

\begin{abstract}
Tadalafil is a phosphodiesterase 5 inhibitor used orally as solid dosage form. The suspension of this drug has been used for pulmonary arterial hypertension treatment in pediatrics. The aim of this work was to investigate the influence of non-ionic surfactant (Tween 80) on the physical characteristics, drug particle size, and stress-shear rate rheogram of tadalafil $0.1 \%$ suspension. Several formulations prepared by xanthan gum as suspending agent. Glycerin has been used as wetting agent. The several amounts of Tween 80 were added and the characteristics of suspensions were evaluated during 6 months. The results showed the effect of surfactant on sediment volume and resuspendibility. The particle size of tadalafil was affected by surfactant amount. This result showed an optimum effect of Tween 80 on drug particle size. The viscosity behavior evaluation of tadalafil $0.1 \%$ suspension showed Tween 80 effect. This study showed that Tween 80 can dramatically affect viscosity of suspensions. The results of this study have demonstrated the effect of Tween 80 on physical properties of tadalafil $0.1 \%$ oral suspension. An ideal drug particle size was observed in particular amount of Tween $80(0.15 \% \mathrm{w} / \mathrm{v})$.
\end{abstract}

Keywords: Tadalafil, suspension, Tween 80, particle size, rheology

Pharm Biomed Res 2015; 1(2): 35-43

DOI: 10.18869/acadpub.pbr.1.2.35

\section{Introduction}

Many pharmaceutical dosage forms are suspensions of a solid drug with a poor solubility in water in an aqueous medium (1). Suspensions are coarse dispersions of solid particles in a liquid medium, generally water or any aqueous solution with particle size between 0.1-10 $\mu \mathrm{m}$ (2). They are thermodynamically unstable systems and preparation of these dosage forms is often associated with problems of physical stability (3). Sedimentation, aggregation of the single particles leading to "cake formation", difficulty of dispersibility of sediment and crystal growth is the main problems in these systems (4). Pharmaceutical suspensions have to be homogenous and stable for accurate dose administration. These formulations require to avoid cake formation and to maintain the sediment in a flocculate state for easy dispersion before dispensation. Different agents can be used for this purpose: surfactants as wetting agent of the solids, thickeners for rheological adjustment or 
electrolytes to modify the conditions of the electrolytic characteristics of medium (2). Polymers as suspending agents can lead to controlled flocculation of drug particles and adjust the redispersibility of suspensions (5). A surfactant is generally used to disperse the hydrophobic solid particles of drug. Surfactants may give rise either electrostatic or steric stabilization of dispersed systems. An excess amount of surfactant generally use in suspensions, which may be required to improve milling process in industry. However, an excessive amount of these agents may result in micellar depletion flocculation and active ingredient solubilization. The latter may promote destabilization due to crystal growth of drug particles. Hence, quantification of surfactant adsorption is of significant importance to adjust the physical characteristics of formulation of pharmaceutical suspensions (1).

Tadalafil is a poorly water soluble medicine that recently has been taken into consideration for the treatment of pulmonary arterial hypertension in pediatrics (6,7). Pulmonary arterial hypertension $(\mathrm{PAH})$ is a progressive and finally lethal disease that has no cure (8) and presents as an increased blood pressure and vascular resistance in the pulmonary arteries (9).

Pulmonary arterial hypertension in children may be related to variety of underlying disorders and plays a significant role in the morbidity and mortality. It has been reported that children with PAH who do not undergo treatment, survive less than one year. Recent advances in basic pulmonary vascular biology have resulted in new therapies for this lethal disease, such as Phosphodiesterase-5 inhibitors (PDE5inhibitors) like tadalafil (10).

The lack of a suitable dosage forms for children, endangers $40 \%$ of the world's population by the risk of preventable adverse effects, suboptimal dose, nonadmission, and unavailability of new medicines $(11,12)$. Many drugs already prescribed in pediatrics have not been sufficiently studied in scientific trials and have not been approved for the use in pediatrics in particular. However because of the lack of more effective and safer alternatives, these medications can still be prescribed in pediatrics as "off-label" $(6,13)$.

Because of the absence of suitable pediatric dosage form, in these cases tadalafil prepared and administered as extemporaneously suspensions by grinding its tablets and mixing it with water and other excipients (14). The general problems of these methods are the possibility of errors in dosing, the probability of presence of excipients in adult pharmaceutical dosage forms that are not suitable for children, limitations on the manipulation of enteric-coated tablets, multi-layer tablets with non-homogeneous matrix and controlled-release tablets. Some excipients may be added during the process of manipulating that the active ingredients or the other components are not compatible with. For example, incompatibility of primary amines such as amlodipine with reducing sugars such as lactose. Also, since the adult dosage forms probably already contain excipients, adding excipients during manipulation should not exceed the limitations unnecessarily $(9,15)$.

The aim of this work was to study the effect of formulation ingredients, especially Tween 80 as surfactant on stability and physical characteristics of Tadalafil $0.1 \%$ oral suspension.

\section{Materials and methods}

Materials

Tadalafil was donated by Osvah Pharmaceutical Co. (Tehran, Iran). Tween 80 , sodium benzoate, and glycerin were 
purchased from Scharlau Co. (Spain); Methyl paraben and dimethyl sulfoxide were received from Merck Co. (Germany); Xanthan gum was purchased from Titrachem Co. (Iran); and sucrose was prepared in bulk.

\section{Preparation of suspensions}

All of suspensions contain $0.1 \%(\mathrm{w} / \mathrm{v})$ of tadalafil (Table 1). For the preparation of suspensions, the xanthan gum, and Tween 80 were initially dispersed in water containing sugar and the preservative agents. After two days xanthan gum was thoroughly hydrated. Then this blend was stirred by a double bladed electric mixer (IKA®-WERKE, Germany) until became totally dispersed, then tadalafil which was wetted by glycerin was added to the vehicle and dispersed with the electric mixer by stirring $300 \mathrm{rpm}$ for 10 minutes. The suspensions were transferred to the $25 \mathrm{~mL}$ stoppered graduated glass cylinders and stored at room temperature under static conditions.

\section{Evaluation of suspensions}

\section{Rheological assessment}

For each suspension, viscosity (cP) was determined at the angular velocity of $0,0.5$, $1,2,2.5,4,5,10,20,50$, and $100 \mathrm{rpm}$, using a Brookfield (DV-II+ viscometer, USA) rotary viscometer with RV7 spindle.

\section{Sedimentation volume}

The sedimentation volume ( $\mathrm{F}$ factor) was measured in $25 \mathrm{~mL}$ graduated glass cylinders. Each suspension was shaken to ensure uniform dispersion prior to the sedimentation test. The sedimentation volume was recorded at $0,30,90$, and 180 day storage period for four samples of each formulation.

\section{Resuspendability}

The resuspendability of suspensions was evaluated qualitatively for four samples of each suspension. The test was performed by shaking the cylinder manually at $180^{\circ}$ movements after sedimentation was completed. Based on the number of shaking required to convert the sediment to uniformly dispersed suspension the formulations were evaluated.

\section{Particle size determination}

Particle size of all formulations was determined after 30, 90 and 180 days quantitatively using a particle size analyzer (Shimadzu SA-CP3 particle size analyzer, Japan). Also, the growth of crystalline particles in each sample was evaluated qualitatively at the end of the period using an optical microscope Nikon Eclipse TS 100.

Assay

Tadalafil is practically insoluble in water, but freely soluble in dimethyl sulfoxide (16). Several dilutions $(5,10,15,20$, and 30 $\mu \mathrm{g} / \mathrm{ml})$ were prepared and the UV absorbance was measured in $\lambda_{\max }=284 \mathrm{~nm}$ (17) by Varian Cary 50 UV-Visible spectrophotometer (Australia). $5 \mathrm{~mL}$ of each suspension was dissolved in solvent and then filtered several times for complete elimination of waste material. Then remaining solution transferred to a $100 \mathrm{~mL}$ volumetric flask. The absorbance of each sample was determined by UV-Visible spectrophotometer and the process was repeated 3 times for each sample. Concentrations corresponding to absorptions were calculated using standard curve $($ Abs $=0.0338$ Conc -0.0042 , $\left.r^{2}=0.9998\right)$.

\section{Statistical Analysis}

Student t-test and ANOVA followed Tukey test were used to determine significant 
differences beTween groups and " $P<0.05$ " was considered significant.

\section{Results}

Table 1 shows the ingredients' amount of 13 formulations. F1-F9 showed no suitable physical characteristics (data were not shown), so 5 formulations (F9-F13) were studied for 6 months. The assay showed that all of the suspensions were in pharmacopeial criteria $(97.20 \pm 1.08,98.64 \pm 0.62,99.14 \pm$ $0.48,101.50 \pm 1.02$, and $98.86 \pm 0.74$, respectively).

The results of F-value and resuspendibility of selected formulations (Table 2) showed that these factors in formulations containing Tween 80 was better than F9 $(P<0.05)$. The results proved that there is no significant change in F-value and resuspendibility in formulations containing several amounts of Tween 80 ( $P=0.105)$. The presence of Sodium benzoate as microbial preservative changed the F-factor after 6 months $(P=$ 0.004). This effect was observed in resuspendibility too.

The results of particle size analyzing are shown in table 2 and figure 1 . The particle size decreased in formulations containing Tween $80(P<0.001)$. The optimum of particle size was observed in F12 with $0.15 \%$ Tween $80 \quad(P<0.01)$. Sodium benzoate decreased tadalafil particle size in formulation 13 in comparison with $\mathrm{F} 12(P=$ 0.0324).

Figure 2 shows the rheological characteristics of tadalafil suspensions. The lowest viscosity was observed in F9 with no surfactant. The ideal viscosity was observed in F12 and F13.

\section{Discussion}

In this research the effect of several amounts of Tween 80, as surfactant, on physical stability of tadalafil $0.1 \%$ suspension was evaluated. Surfactants are bipolar molecules with hydrophilic polar head and hydrophobic nonpolar tail which in most of cases the hydrophobic tail made up of a hydrocarbon section linked to the polar region (18). Surfactants can be cationic, anionic, amphoteric, or nonionic (19). They form micelles in solutions, which plays a significant role in their properties like solubilizing ability. In polar solutions like water, small non-polar molecules will be solubilized in the hydrophobic core of the micelles (20).

Surfactants are utilized as wetting agent, foaming agent, corrosion inhibitor, bactericide, antistatic agent, dispersant, enhancer for percutaneous absorption, flocculating agent, in suppository bases, water based or suspension aerosols, hard gelatin capsules, in respiratory distress therapy, in microbiology, or to make membrane, liquid crystal, micro emulsion, liposome, vesicle or gel, in addition to their usual use as soap, detergent, emulsifier, and solubilizer $(21,22)$.

The results of physical evaluation showed that $\mathrm{F}$-value was increased in formulations containing Tween 80. Surfactants such as Tween 80 improve the wettability of the solid particles in a suspension and lower the surface tension of the continuous phase which inhibits the floating of the particle and decreases aggregation, so sediment volume increases (23-25). In contrast, cationic surfactants lead to coagulation of finely dispersed suspensions because of stability loss due to neutralization of surface charge of particles and flocculating effect of the cationic surfactants (26).

Rudnev et al. reported that the stability of the suspension containing the Calcium Hydroxyapatite (HAP) particles relies on the concentration of surfactant in solution, which enhanced considerably at Tween 80 concentrations above $3.0 \times 10^{-3} \mathrm{M}$ (27).

The particle size of tadalafil was affected by surfactant amount. This result showed an optimum effect of tweeon 80 on drug particle size. The particle sized increased by 
Table 1 Composition of Tadalafil $0.1 \%$ formulations

\begin{tabular}{|c|c|c|c|c|c|c|c|c|}
\hline \multirow{2}{*}{$\begin{array}{l}\text { Formulation } \\
\text { code }\end{array}$} & \multicolumn{8}{|c|}{ Composition of ingredients (g) } \\
\hline & $\begin{array}{c}\text { Xanthan } \\
\text { gum }\end{array}$ & Glycerin & Tadalafil & $\begin{array}{c}\text { Methyl } \\
\text { paraben }\end{array}$ & $\begin{array}{c}\text { Sodium } \\
\text { benzoate }\end{array}$ & $\begin{array}{c}\text { Tween } \\
80\end{array}$ & Sucrose & $\begin{array}{c}\text { Water* } \\
(\mathrm{ml})\end{array}$ \\
\hline F1 & 1 & 20 & 0.1 & 0.1 & - & - & - & to 100 \\
\hline F2 & 0.5 & 20 & 0.1 & 0.1 & - & - & - & to 100 \\
\hline F3 & 0.25 & 20 & 0.1 & 0.1 & - & - & - & to 100 \\
\hline F4 & 0.15 & 20 & 0.1 & 0.1 & - & - & - & to 100 \\
\hline F5 & 0.25 & 20 & 0.1 & 0.1 & - & 0.25 & - & to 100 \\
\hline F6 & 0.25 & 20 & 0.1 & 0.1 & - & 0.5 & - & to 100 \\
\hline F7 & 0.25 & 20 & 0.1 & 0.1 & - & 0.15 & - & to 100 \\
\hline F8 & 0.25 & 20 & 0.1 & - & 0.1 & 0.15 & - & to 100 \\
\hline F9 & 0.25 & 20 & 0.1 & 0.1 & - & - & 30 & to 100 \\
\hline F10 & 0.25 & 20 & 0.1 & 0.1 & - & 0.25 & 30 & to 100 \\
\hline F11 & 0.25 & 20 & 0.1 & 0.1 & - & 0.5 & 30 & to 100 \\
\hline F12 & 0.25 & 20 & 0.1 & 0.1 & - & 0.15 & 30 & to 100 \\
\hline F13 & 0.25 & 20 & 0.1 & - & 0.1 & 0.15 & 30 & to 100 \\
\hline
\end{tabular}

Table 2 Result of evaluation of sedimentation volume, resuspendability, and particle size of evaluated suspension $(\mathrm{n}=4)$

\begin{tabular}{|c|c|c|c|c|c|c|c|c|c|}
\hline \multirow{3}{*}{$\begin{array}{l}\text { Formulation } \\
\text { code }\end{array}$} & \multicolumn{9}{|c|}{ Characteristics of evaluated tadalafil suspensions } \\
\hline & \multicolumn{3}{|c|}{$1^{\text {st }}$. month } & \multicolumn{3}{|c|}{$3^{\text {rd }}$. month } & \multicolumn{3}{|c|}{$6^{\text {th }}$. month } \\
\hline & 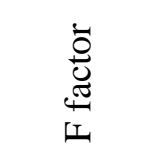 & 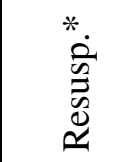 & 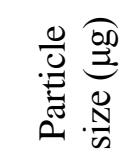 & 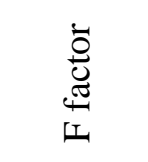 & 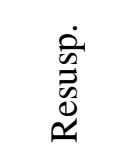 & 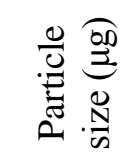 & 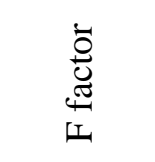 & 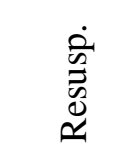 & 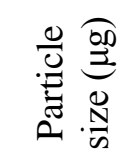 \\
\hline F9 & $0.97 \pm 0.2$ & $1.5 \pm 0.06$ & $31.6 \pm 0.5$ & $0.99 \pm 0.02$ & $1.0 \pm 0.0$ & $39.4 \pm 0.1$ & $0.95 \pm 0.04$ & $1.75 \pm 0.5$ & $40.8 \pm 0.1$ \\
\hline F10 & $0.98 \pm 0.03$ & $1.25 \pm 0.5$ & $8.09 \pm 0.2$ & $0.99 \pm 0.02$ & $1.0 \pm 0.0$ & $8.9 \pm 0.2$ & $0.99 \pm 0.02$ & $1.25 \pm 0.5$ & $8.6 \pm 0.9$ \\
\hline F11 & $1.0 \pm 0.0$ & $1.0 \pm 0.0$ & $8.4 \pm 0.2$ & $1.0 \pm 0.0$ & $1.0 \pm 0.0$ & $6.5 \pm 0.4$ & $0.99 \pm 0.05$ & $1.0 \pm 0.0$ & $8.0 \pm 0.1$ \\
\hline F12 & $0.94 \pm 0.04$ & $2.5 \pm 1$ & $1.4 \pm 0.3$ & $0.96 \pm 0.02$ & $1.5 \pm 0.6$ & $3.2 \pm 0.1$ & $0.99 \pm 0.02$ & $1.25 \pm 0.5$ & $5.9 \pm 0.5$ \\
\hline F13 & $0.95 \pm 0.03$ & $2.25 \pm 0.5$ & $4.9 \pm 0.1$ & $0.99 \pm 0.02$ & $1.25 \pm 0.5$ & $4.8 \pm 0.6$ & $0.47 \pm 0.15$ & $5.5 \pm 1.3$ & $4.9 \pm 0.2$ \\
\hline
\end{tabular}



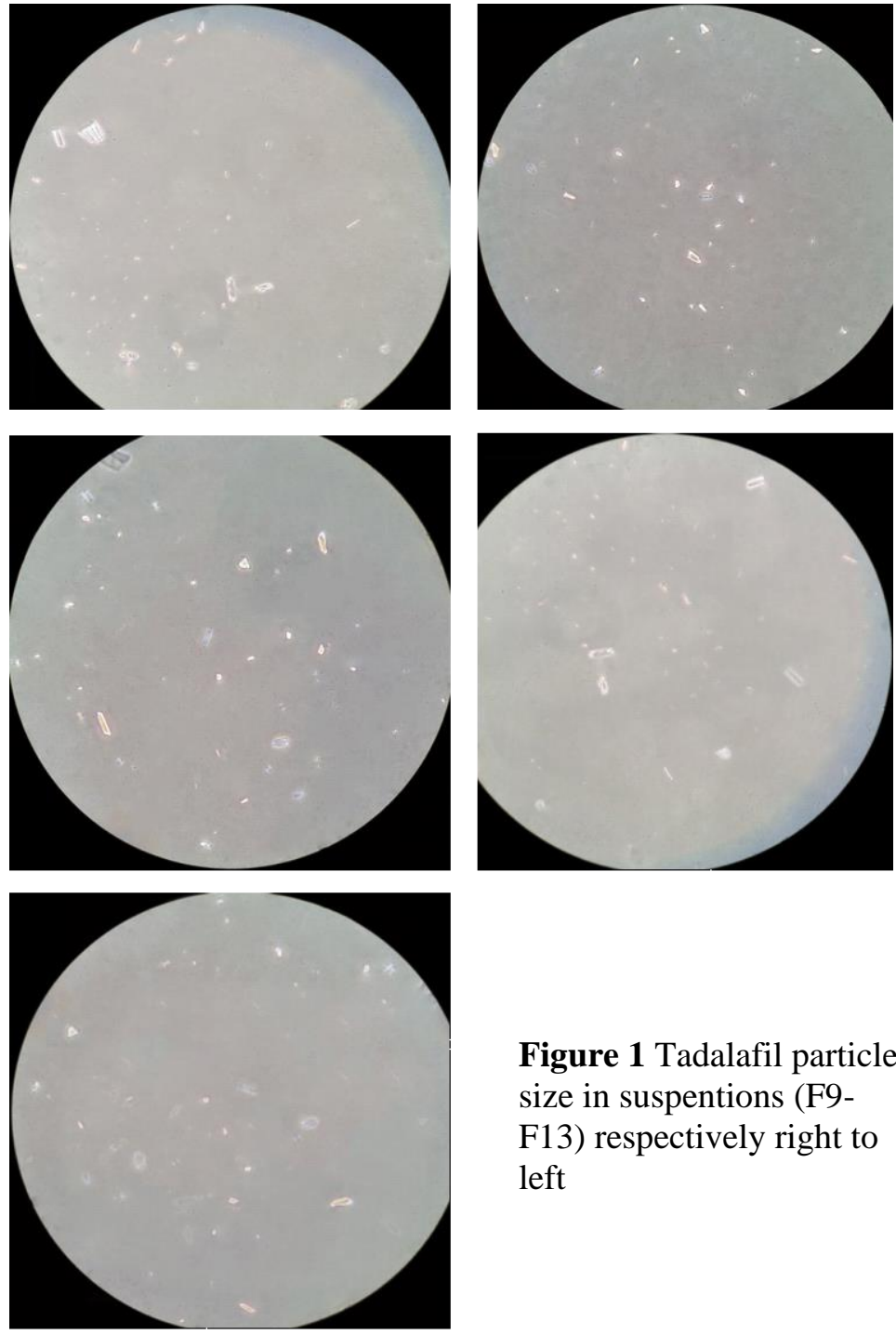

Figure 1 Tadalafil particle size in suspentions (F9F13) respectively right to left

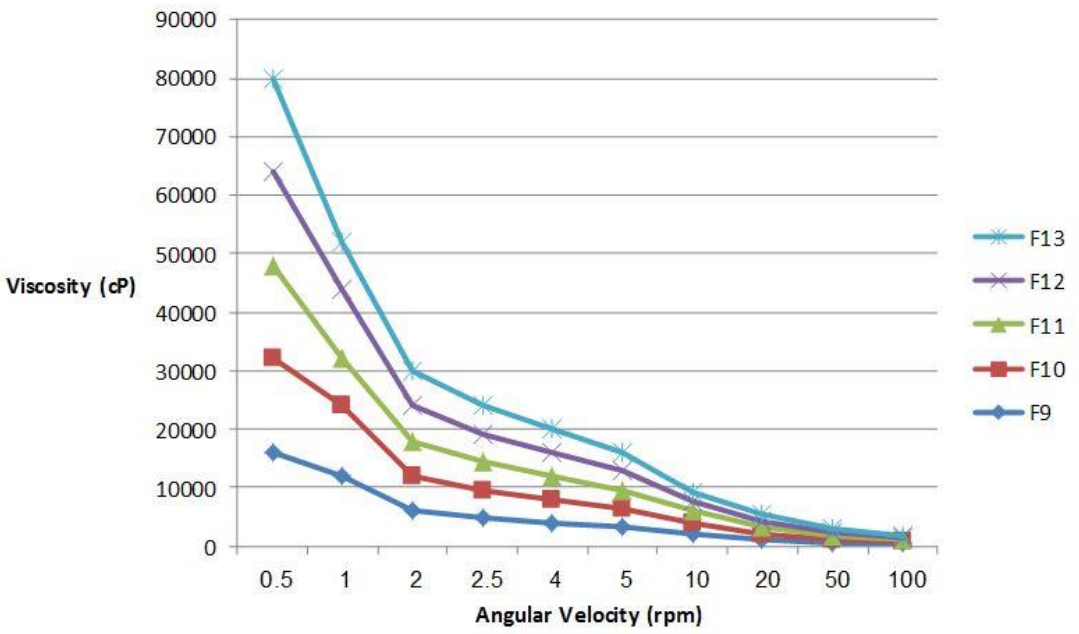

Figure 2 Results of rheology study 
increasing in amount of Tween 80 . In one study, effect of concentration of Tween 80 on particle size and zeta potential of diclofenac sodium-loaded chitosan nanoparticles was evaluated. The results showed that particle size increases along with increasing concentration of Tween 80 . Inversely, zeta potential decreases in higher concentration of Tween 80. This might be because of amphiphilic property of Tween 80 which deposited at the particle surface and caused increasing in particle size. Further, it could protect surface charge of the diclofenac-loaded nanoparticles led to decrease in zeta potential. Sedimentation rate of diclofenac-loaded nanoparticles, confirmed this expectation. This study proved that Tween 80 at the concentration of $0.02 \% \mathrm{w} / \mathrm{v}$ could be considered as a suitable concentration for stabilization of diclofenac nanoparticle system (28).

A study on stability of titanium dioxide suspensions presented that the zeta potential and aggregation stability of the suspensions was not affected significantly by the nonionic surfactant. The $\mathrm{pH}$ of dispersion media showed considerably effect on influence of ionic surfactants on the aggregation stability of the suspensions. At $\mathrm{pH}$ values above the isoelectric point of titanium dioxide suspensions $\left(\mathrm{pH}_{0}=6.2\right)$, the suspensions showed a high aggregation stability in the presence of the sodium dodecylbenzene sulfonate, as anionic surfactant, (irrespective of its content), while, at $\mathrm{pH}<\mathrm{pH}_{0}$, the aggregation stability of the suspensions significantly increases with the surfactant concentration. In the presence of the benzethonium chloride, as cationic surfactant, the aggregation stability of the suspensions is independent of the surfactant concentration at $\mathrm{pH}<\mathrm{pH}_{0}$, whereas, at $\mathrm{pH}>\mathrm{pH}_{0}$, it increases with the surfactant concentration (29).
The viscosity behavior evaluation of tadalafil $0.1 \%$ suspension showed Tween 80 effect. A study showed that Tween 80 can dramatically affect viscosity of suspensions and increase the yield stress as well as the plastic viscosity. Adding surfactant to suspensions also changed the rheograms of them. The rheogram of surfactant-free suspension presented minimum thixotropy, TI was $15.8 \%$. However, the addition of $0.5 \% \mathrm{w} / \mathrm{w}$ Tween 80 during hydration increased the TI to $30.6 \%$. Increasing the concentration of surfactant caused an increase in the peak shear stress (30).

In another study, the effect of surfactants on shear-thickening behavior of concentrated polymer dispersions was evaluated. The results showed that the shear-thickening behavior was influenced by surfactants. This occurs as a result of ability of surfactants in changing the surfaces forces and inter-particle forces. Various kinds of surfactants, such as cationic, anionic, nonionic, and amphoteric surfactants were added to the shear-thickening fluid and their rheological properties were measured. The results proved that all kinds of surfactants could enhance shear-thickening behavior by changing their electrostatic, steric, or lubrication interaction (31). Recently, in one study the effect of several factors on stability of tadalafil nanosuspension was evaluated. Tween 80 and span 80 were used for this formulation. The surfactant mixture aided the production of nanosuspensions with an average particle size of $193 \pm 8 \mathrm{~nm}$ and with short-term stability sufficient for further processing (32).

\section{Conclusion}

This study has demonstrated the effect of Tween 80 on physical properties of tadalafil $0.1 \%$ oral suspension. An increase concentration of surfactant increased sediment volume of drug. An ideal drug 
particle sized was observed in particular amount of Tween $80(0.15 \% \quad \mathrm{w} / \mathrm{v})$. The rheological behavior of suspension was affected by surfactant amount too.

\section{Acknowledgment}

This work was supported by a grant from the research council of the Mazandaran University of Medical Sciences.

\section{References}

1. Zhu W, Sabatino B, Govoreanu R, Verbruggen K, Martin JC, Van der Meeren P. Preferential adsorption of polysorbate 20 molecular species in aqueous paliperidone palmitate suspension. Colloid Surf A: Physicohem Eng Aspect 2011;384:691-7.

2. Nielloud F, Marti-Mesters G, Fortune R, Mestres JP, Maillols H. Formulation of insoluble drugs, study of pharmaceutical suspensions by response surface methodology. Anal usis 1998;26:277-81

3. Saeedi M, Dallalpour-Mohammadi N, Farid Dj. Prevention of crystal growth in acetaminophen suspensions by the use of PVP and bovine serum albumin. Daru 2003;11:106-14.

4. Sha Z, Palosaari S. Mixing and crystallization in suspensions. Chem Eng Sci 2000;55:1797-806.

5. Ntawukulilyayo JD, De Smedt SC, Demeester J, Remon JP. Stabilition of suspensions using sucrose esters and low substituted n-octenyl succinate starchxanthan gum associations. Int J Pharm 1996;128:73-9.

6. Oudiz RJ, Brundage BH, Galiè N, Ghofrani HA, Simonneau G, Botros FT, Chan M, Beardsworth A, Barst RJ. Tadalafil for the treatment of pulmonary arterial hypertension: A double-blind 52-week uuncontrolled extension study. J Am Coll Cardiol 2012;60:768-74

7. Falk JA, Philip KJ, Schwarz ER. The emergence of oral tadalafil as a once-daily treatment for pulmonary arterial hypertension. Vasc Health Risk Manag 2010;6:273-80.

8. Buckley MS, Staib RL, Wicks LM, Feldman JP. Phosphodiesterase-5 inhibitors in management of pulmonary hypertension: safety, tolerability, and efficacy. Drug Health Patient Saf 2010;2:151-61

9. Barst RJ, Brundage BH, Sundin DP, Oudiz RJ, Simonneau G, Galiè N, Beardsworth A, Ghofrani HA. Tadalafil monotherapy and as add-on to background bosentan in patients with pulmonary arterial hypertension. J Heart Lung Transplant 2011;30:63243.

10. Roofthooft MT, van Loon RL, Berger RM. Management of pulmonary arterial hypertension in children. Paediatr Respir Rev 2010;11:240-5.

11. Milne CP, Bruss JB. The economics of pediatric formulation development for off-patent drugs. Clin Ther 2008:30:2133-45.

12. Breitkreutz J. European perspectives on pediatric formulations. Clin Ther 2008;30:2146-54.

\section{Conflict of interest}

The authors declared no potential conflict of interest with respect to the authorship, and/or publication of this study.
13. Freed AL, Silbering SB, Kolodsick KJ, Rossi DT, Mahjour M, Kingsmill CA. The development and stability assessment of extemporaneous pediatric formulations of Accupril. Int J Pharm 2005;304:13544.

14. Pettit RS, Johnson CE, Caruthers RL. Stability of an extemporaneously prepared tadalafil suspension. Am J Health Syst Pharm 2012;69:592-4.

15. Standing JF, Tuleu C. Paediatric formulationsGetting to the heart of the problem. Int J Pharm 2005;300:56-66.

16. Anonymus, Tadalafil. In: European pharmacopeia. Council Of Europe, $7^{\text {th }}$ Ed. 2012; pp. 4379-4381. E.D.Q.M.

17. Yunoos M, Sankar DG, Kumar BP, Hameed S. UV Spectrophotometric method for estimation of tadalafil in bulk and tablet dosage form. E-J Chem 2010;7:8336.

18. Sriraviteja N, Kumar K, Raju B, Madhuri K. Effect of application of solubilizers such as PVP K 30,PEG 400 and Tween 80 on the enhancement of solubility of ibuprofen by factorial design. IJPRBS. 2013;2:182203.

19. Schramm LL, Stasiuk EN, Marangoni DG. Surfactants and their applications. Annu Rep Prog Chem 2003; 99:3-48.

20. Rangel-Yagui CO, Pessoa AJ, Tavares LC. Micellar solubilization of drugs. J Pharm Pharmaceut Sci 2005;8:147-63.

21. Salager JL. Surfactants Types and Uses. FIRP Booklet \# E300-A. Universidad de los Andes Inc, 2002; p. 3.

22. Mishra M, Muthuprasanna P, Prabha KS, Sobhita Rani PS, Satish Babu IA, Chandiran IS, Arunachalam G, Shalini S. Basics and potential applications of surfactants, A review. Int $\mathrm{J}$ Pharm Tech Res 2009;1:354-65

23. Buhler V. Pharmaceutical technology of BASF excipients. BASF the chemical company Inc. $3^{\text {rd }}$ revised edition. 2008; p. 125

24. Attwood D, Florence AT. Surfactants. In: Physical pharmacy. Pharmaceutical Press, $2^{\text {nd }}$ ed., 2012; p. 50.

25. Somasundaran P, Xiang Y, Krishnakumar S. Role of conformation and orientation of surfactants and polymers in controlling flocculation and dispersion of aqueous and non-aqueous suspensions. Colloids Surf A: Physicochem Eng Aspects 1998;133:125-33.

26. Mikhailova IV, Gerashchenko II. Stability and adsorption properties of suspensions of finely 
dispersed silica in the presence of cationic surfactants. Colloid Journal 2002;64:583-7.

27. Rudnev AV, Ivanova NI, Vanifatova NG, Dzherajan TG. The effect of ultrasonic treatment on the stability of a dispersed system of calcium hydroxyapatite in an aqueous solution of Tween 80. Moscow Uni Chem Bull 2014;69:175-9.

28. Asasutjarit R, Sorrachaitawatwong C, Tipchuwong N, Pouthai S. Effect of formulation compositions on particle size and zeta potential of diclofenac sodiumloaded chitosan nanoparticles. Int J Med Health Pharmaceut Biomed Eng 2013;7:254-6.

29. Petryshyn RS, Yaremko ZM, Soltys MN. Effects of surfactants and $\mathrm{pH}$ of medium on zeta potential and aggregation stability of titanium dioxide suspensions. Colloid J 2010;72:517-22.
30. Kennedy RA, Kennedy ML. Effect of selected nonionic surfactants on the flow behavior of aqueous veegum suspensions. AAPS Pharm Sci Tech 2007;8:171-6.

31. Fang Y, Wei Z, Zhiyuan W, Shouhu X, Wanquan J, Qian C, Xinglong G. Influence of surfactants on shearthickening behavior in concentrated polymer dispersion. J Nanopart Res 2013;15:21-2.

32. Obeidat WM, Sallam AA. Evaluation of tdadlafil nanosuspensions and their PEG solid dispersion matrices for enhancing its dissolution properties. AAPS Pharm Sci Tech 2014; 15:364-74. 